\title{
Time-resolved study of intervalence band thermalization in a GaAs quantum well
}

\author{
A. M. T. Kim, S. Hunsche, a) T. Dekorsy, and H. Kurz \\ Institut für Halbleitertechnik II, Rheinisch-Westfälische Technische Hochschule (RWTH) Aachen, \\ Sommerfeldstr. 24, D-52056 Aachen, Germany \\ K. Köhler \\ Fraunhofer Institut für Angewandte Festkörperphysik, 79108 Freiburg, Germany
}

\begin{abstract}
The thermalization of optically excited cold holes in a GaAs quantum well is investigated by femtosecond two-color pump-probe measurements. Clear evidence is found for scattering from heavy-holes into the lowest light-hole band due to LO-phonon absorption. We obtain firm data on scattering times which depend strongly on lattice temperature. They vary from $230 \mathrm{fs}$ at room temperature to $900 \mathrm{fs}$ at $T=105 \mathrm{~K}$. The experimental data are well reproduced by numerical calculations.
\end{abstract}

Firm experimental data on ultrafast carrier dynamics in semiconductors and semiconductor heterostructures are of prime importance for the development of novel ultrafast opto-electronic devices. The development of ultrashort-pulse lasers has stimulated a large number of time-resolved studies addressing the picosecond and subpicosecond dynamics of nonequilibrium carriers in GaAs and other III-V semiconductors. ${ }^{1,2}$ Since the density of states in the conduction band is generally much lower than that of the valence band, in most studies the experimental signals are dominated by the dynamics of optically excited electrons. ${ }^{3}$ Only in a few specific experiments could information on the hole relaxation dynamics be obtained in special sample structures. ${ }^{4-8}$ In these studies, holes were excited with a certain excess energy and subsequent cooling processes were observed. In contrast, optical excitation of excitons close to the band edge will create initially cold holes. In this case, the hole dynamics at elevated lattice temperatures is expected to be dominated by the absorption of longitudinal optical (LO) phonons that leads to a heating of the initial hole distribution. ${ }^{3,7}$

In this letter, we investigate the intervalence band thermalization of optically excited holes. Femtosecond timeresolved measurements of the bleaching of the heavy-hole (HH) and light-hole (LH) exciton transition in a GaAs multi quantum well (MQW) sample are performed. The nonlinear absorption of the excitonic transitions is determined by two contributions, namely the reduction of the oscillator strength and the broadening of the exciton line. ${ }^{9}$ It has been shown previously that the reduction of the oscillator strength strongly depends on the subband occupation number, while the intrasubband carrier distribution does not play a significant role. ${ }^{9,10}$ In the same work it was found that broadening is not affected by changes of the subband occupation. Furthermore, measurements of absorption changes at the lowenergy edge of the E1H1 transition-which are dominated by broadening - under various excitation conditions only show an "instantaneous" signal without any further dynam-

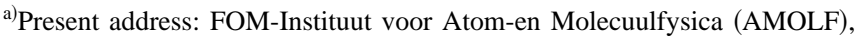
Kruislaan 407, 1098 SJ Amsterdam, The Netherlands.
}

ics on a picosecond time scale, indicating that this signal contribution is not sensitive to the exact intrasubband carrier distribution, either. ${ }^{11}$ These facts make it possible to measure changes of the hole subband occupation by choosing sample and excitation parameters that prevent any electron intersubband dynamics.

Our experiments are performed in a two-color pumpprobe scheme, using femtosecond pulses of a Kerr-lens mode-locked Ti:sapphire laser with a pulse duration of $50 \mathrm{fs}$ and a bandwidth of $\approx 38 \mathrm{meV}$ full width at half-maximum (FWHM). In order to allow selective excitation and probing of various excitonic transitions, the pump pulses are spectrally filtered with interference filters of $\approx 9 \mathrm{meV}$ FWHM, and the probe energy is selected using a monochromator $(\approx 2 \mathrm{meV}$ FWHM) behind the sample. The reduction of the bandwidth results in a temporal broadening of the pump pulses to $\approx 300 \mathrm{fs}$, while spectrally filtering the probe pulse behind the sample does not lead to a further limitation of the temporal resolution. We measure optically induced transmission changes of a GaAs MQW sample by using a photomultiplier behind the monochromator and applying a lock-in technique. The sample consists of 40 periods of $6.5 \mathrm{~nm}$ GaAs wells and $20 \mathrm{~nm} \mathrm{Al}_{0.35} \mathrm{Ga}_{0.65}$ As barriers grown by molecular beam epitaxy. The substrate is removed by chemical etching to allow transmission measurements. The sample is placed in a closed-cycle cryostat to perform measurements at various lattice temperatures.

The optically induced transmission changes near the band edge of the MQW sample are completely dominated by changes of the lowest two excitonic absorption lines. As illustrated in Fig. 1 they correspond to transitions between the first $\mathrm{HH}$ and LH subbands and the lowest electron subband. In the same figure a typical differential transmission spectrum is shown obtained in a "white-light" pump-probe experiment ${ }^{3,9}$ at room temperature taken 3 ps after resonant excitation at the E1H1 transition. The spectrum reflects a quasi-equilibrium situation. Within this time interval the optically created carriers are completely thermalized with the lattice. The splitting between the $\mathrm{HH}$ and $\mathrm{LH}$ excitons of 25 $\mathrm{meV}$ is large enough to allow selective pumping and probing. Bleaching of continuum transitions can be neglected in 


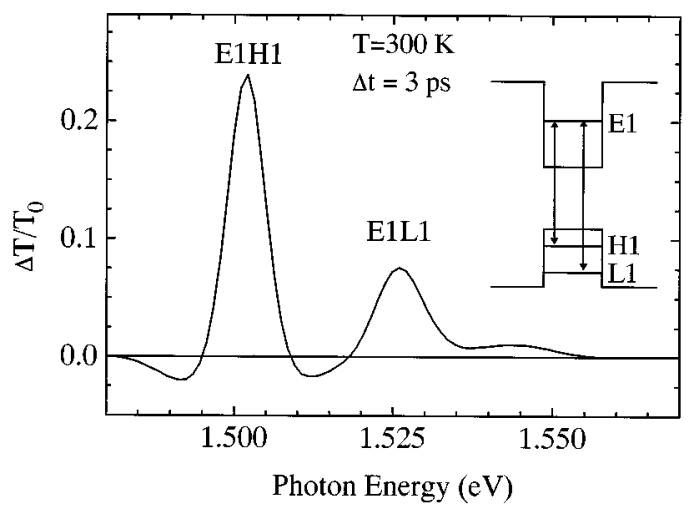

FIG. 1. Room temperature differential transmission spectrum of the MQW sample at a time delay of $3 \mathrm{ps}$. The total carrier density is $N_{\text {exc }}=1.1 \times 10^{11} \mathrm{~cm}^{-2}$. The peaks correspond to the bleaching of heavy hole and light hole exciton transitions.

comparison with the transmission changes at the exciton peaks.

Figure 2 shows the temporal evolution of the induced transmission changes at room temperature after resonant excitation of the $\mathrm{HH}$ and $\mathrm{LH}$ exciton, respectively. The transients were measured with the probe tuned to the maxima of the exciton lines, as indicated in the figure. The total carrier density for both excitation wavelengths is $N_{e x c}=1.1 \times 10^{10}$ $\mathrm{cm}^{-2}$, as estimated from the pump power and the spot size. For the case of LH excitation, the time dependence of the $\mathrm{HH}$ and LH transmission changes is characterized by a rise that closely follows the integral of the pump pulse and does not show any indication of carrier relaxation on a picosecond time scale. In contrast, after $\mathrm{HH}$ excitation, we observe an initially enhanced bleaching at the $\mathrm{HH}$ transition, which relaxes within $\approx 1$ ps. Complementary to this relaxation, the rise of the LH bleaching is significantly slower than under LH excitation.

While the "overshoot" of the HH bleaching is very similar to early results from Knox et al., who studied the ionization of excitons by LO phonon absorption, ${ }^{12}$ the delayed increase of the LH bleaching indicates that the transient bleaching dynamics in our experiment cannot be ex-

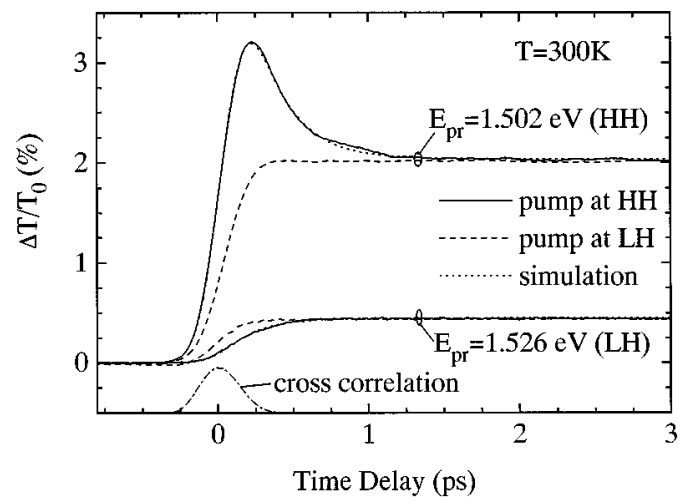

FIG. 2. Time evolution of the pump induced transmission change measured at the maxima of $\mathrm{HH}$ and $\mathrm{LH}$ lines. The pump was tuned either to the $\mathrm{HH}$ line (solid lines) and the LH line (dashed lines). The excitation density for both pump energies is $N_{e x c}=1.1 \times 10^{10} \mathrm{~cm}^{-2}$. Results of numerical simulation are also shown (dotted lines). plained by exciton ionization alone. In fact, the transmission signature after $\mathrm{HH}$ excitation matches the expected dynamics associated with interband population changes of cold holes which are heated up to lattice temperature by LO phonon absorption: Under the chosen experimental conditions, only heavy holes are optically excited. Due to thermalization with the lattice however, a significant number of holes will be transferred into the $\mathrm{LH}$ band since the $\mathrm{L}-\mathrm{H}$ splitting is on the order of the thermal energy of the lattice. This interpretation is supported by a numerical simulation based on coupled rate equations for the $\mathrm{HH}$ and $\mathrm{LH}$ occupation. The simulations include both an "instantaneous" contribution due to broadening and electron subband occupation and a contribution due to the HH and LH subband occupation changes. The simulation results are indicated by the dotted curves in Fig. 2 , which have been obtained assuming a single intervalence band scattering time of $\tau_{H-L}=230 \mathrm{fs}$. Obviously, the experimental data can be closely reproduced by the simulation, indicating a common cause for the $\mathrm{HH}$ bleaching decay and the delayed LH bleaching increase.

Although the experimental situation is more complicated, no significant changes of the relative $\mathrm{LH}$ and $\mathrm{HH}$ occupations can be observed in the experimental data after LH excitation. Due to the HH continuum both heavy and light holes will be excited so that intervalence band thermalization may also occur by hole-hole scattering processes. As the $\mathrm{L}-\mathrm{H}$ splitting is smaller than the $\mathrm{LO}$ phonon energy, $\mathrm{L} \rightarrow \mathrm{H}$ transitions can only occur by emission of acoustic phonons or absorption of LO phonons. The former process is known to take place on a time scale of $130 \mathrm{ps}^{4}{ }^{4}$ The latter is quite inefficient due to the large momentum change required. We note that the bleaching dynamics after resonant LH excitation does not show any fast changes which could be assigned to ionization of the LH excitons.

In order to further verify our interpretation of the experimental data, we performed measurements at different temperatures. Theory predicts that interband scattering rates depend linearly on the phonon occupation number and therefore on the lattice temperature. ${ }^{13}$ For the case of excitation at the $\mathrm{HH}$ subband minimum the H-L scattering rate due to $\mathrm{LO}$ phonon absorption is given by: ${ }^{13}$

$$
\begin{aligned}
\tau_{H-L}^{-1}= & \frac{e^{2} \omega_{L O}}{8 \pi \epsilon_{0}}\left[\frac{1}{\epsilon_{\infty}}-\frac{1}{\epsilon_{s}}\right] N_{L O} \int \frac{H_{H L}(Q)}{Q} \delta_{H L}\left(E_{L}\left(\mathbf{k}_{\mathbf{L}}\right)\right. \\
& \left.-E_{H}(0)-\hbar \omega_{0}\right) d \mathbf{k}_{\mathbf{L}},
\end{aligned}
$$

where $\epsilon_{\infty}=10.92, \epsilon_{s}=12.90, \mathbf{k}_{\mathbf{L}}$ is the final state wavevector of the electron, $Q=\left|\mathbf{k}_{\mathbf{L}}\right|$ is the in-plane phonon-wavevector component, $\omega_{L O}$ is the LO-phonon frequency, $N_{L O}$ is the phonon occupation number:

$$
N_{L O}=\left[\exp \left(\frac{\hbar \omega_{L O}}{k T}\right)-1\right]^{-1},
$$

and $H_{H L}(\mathrm{Q})$ is the multisubband coupling coefficient:

$H_{H L}(Q)=\iint d z_{1} d z_{2} F_{H L}\left(z_{1}\right) F_{H L}\left(z_{2}\right) \exp \left(-Q\left|z_{1}-z_{2}\right|\right)$

where $F_{H L}(z)=F_{H}(z) F_{L}(z)$, with $F_{H}(z), F_{L}(z)$ representing the hole wave functions. 


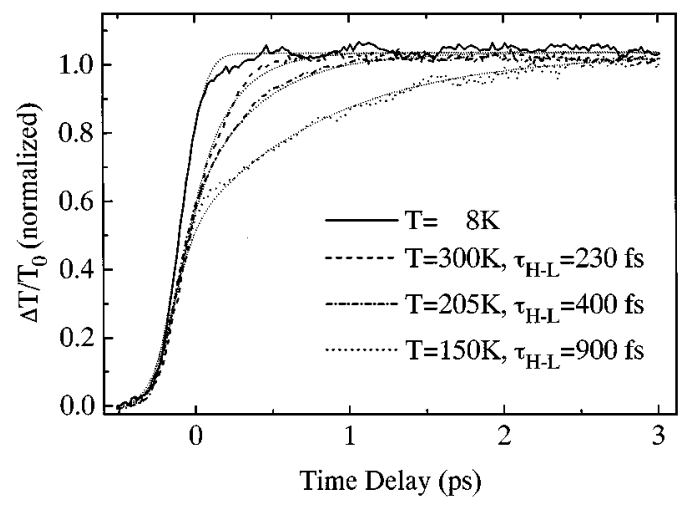

FIG. 3. Time evolution of the transmission changes at the maximum of the LH line after $\mathrm{HH}$ excitation at various temperatures. The short-dotted lines represent numerical simulations of the experimental data.

Figure 3 shows time-dependent transmission changes probed at the $\mathrm{LH}$ exciton line under $\mathrm{HH}$ excitation at lattice temperatures of $300 \mathrm{~K}, 205 \mathrm{~K}, 150 \mathrm{~K}$ and $8 \mathrm{~K}$. Data taken at $250 \mathrm{~K}$ are omitted for sake of clarity of the figure. The transients are normalized to allow a better comparison of the bleaching dynamics. The rise of the LH bleaching becomes slower with decreasing temperature, as expected from Eqs. (1) and (2). The experimental data can be well reproduced by coupled rate equations and a temperature-dependent $\mathrm{H}-\mathrm{L}$ scattering time $\tau_{H-L}$, that varies from $230 \mathrm{fs}$ at $300 \mathrm{~K}$ to 900 fs at $150 \mathrm{~K}$. At $\mathrm{T}=8 \mathrm{~K}$ only the instantaneous signal contribution is observed since the phonon occupation number is nearly zero and phonon absorption cannot take place $\left(\tau_{H-L} \rightarrow \infty\right)$.

For direct comparison with theory, the experimentally determined scattering rates are plotted versus the phonon occupation number in Fig. 4. We estimate an accuracy of \pm 25 fs in the determination of the scattering times, as indicated by the error bars. The dotted line represents a linear fit through the experimental values. The dashed line shows the result of a direct numerical calculation of the scattering rates $1 / \tau_{H-L}$ based on Eqs. (1)-(3). For this calculation parabolic in-plane-dispersion for both $\mathrm{HH}$ and $\mathrm{LH}$ subbands is assumed. The slope of the theoretical curve is $m_{t h}=16.9 \mathrm{ps}^{-1}$ and a linear fit through the experimental data points gives $m_{e x}=13.7 \mathrm{ps}^{-1}$. Given our assumption of parabolic valence subbands, which is a rather crude approximation,,${ }^{14}$ the agreement between data and the calculated scattering rates is quite satisfactory. Better quantitative agreement would require a full numerical treatment taking into account the precise dispersion of the HH and LH subbands.

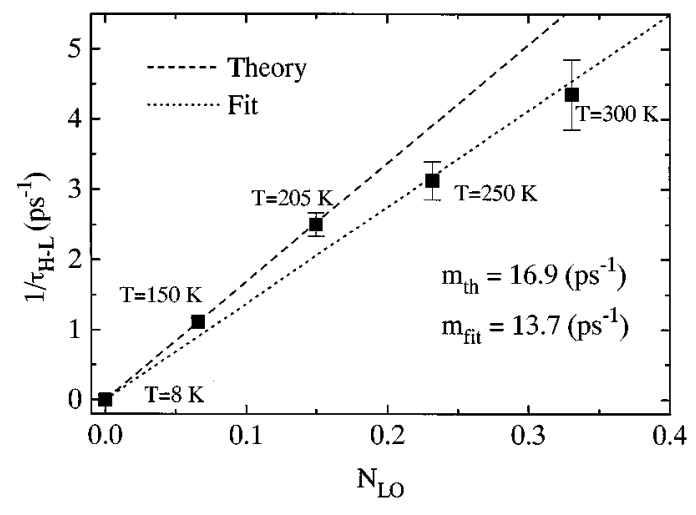

FIG. 4. Scattering rates versus LO-phonon occupation number. The dotted line represents a fit to the experimental data and the dashed line the result of a theoretical calculation.

In conclusion, we present first direct measurements of intervalence band thermalization after optical excitation of cold heavy holes in a quantum well. This thermalization requires a scattering from heavy into light holes which takes place on a subpicosecond time scale by absorption of LO phonons. The experimental data are in very good agreement with theoretical predictions.

The authors gratefully acknowledge R. Lövenich for theoretical support and G.C. Cho for technical assistance. This work was supported by the Volkswagen Foundation.

${ }^{1}$ Optical Nonlinearities and Instabilities in Semiconductors, edited by $\mathrm{H}$. Haug (Academic, Boston, 1988).

${ }^{2} \mathrm{~J}$. Shah, in Spectroscopy of Nonequilibrium Electrons and Phonons, edited by C. V. Shank and B. P. Zakharchenya (Elsevier, Amsterdam, 1992), pp. $57-112$.

${ }^{3}$ S. Hunsche, H. Heesel, A. Ewertz, H. Kurz, and J. H. Collet, Phys. Rev. B 48, 17818 (1993).

${ }^{4}$ R. A. Höpfel, R. Rodrigues, Y. Iimura, T. Yasui, Y. Segawa, Y. Aoyagi, and S. M. Goodnick, Phys. Rev. B 47, 10943 (1993).

${ }^{5}$ A. Tomita, J. Shah, J. E. Cunningham, S. M. Goodnick, P. Lugli, and S. L. Chuang, Phys. Rev. B 48, 5708 (1993).

${ }^{6}$ X. Q. Zhou, K. Leo, and H. Kurz, Phys. Rev. B 45, 3886 (1992).

${ }^{7}$ R. Tommasi, P. Langlot, and F. Vallee, Appl. Phys. Lett. 66, 1361 (1994).

${ }^{8}$ Z. Xu, P. M. Fauchet, C. W. Rella, B. A. Richman, H. A. Schwettman, and G. W. Wicks, Phys. Rev. B 51, 10631 (1995).

${ }^{9}$ S. Hunsche, K. Leo, H. Kurz, and K. Köhler, Phys. Rev. B 49, 16565 (1994).

${ }^{10}$ S. Hunsche, K. Leo, H. Kurz, and K. Köhler, Phys. Rev. B 50, 5791 (1994).

${ }^{11}$ A. M. T. Kim (unpublished).

${ }^{12}$ W. H. Knox, R. L. Fork, M. C. Downer, D. A. B. Miller, D. S. Chemla, and C. V. Shank, Phys. Rev. Lett. 54, 1306 (1985).

${ }^{13}$ Y. L. Lam and J. Singh, IEEE J. Quantum Electron. 31, 923 (1995).

${ }^{14}$ See for example: GaAs and Related Materials, edited by S. Adachi (World Scientific, Singapore, 1994). 